\title{
First Results from the LUX Dark Matter Experiment at the Sanford Underground Research Facility
}

\author{
D. S. Akerib, ${ }^{2}$ H. M. Araújo, ${ }^{4}$ X. Bai, ${ }^{8}$ A. J. Bailey, ${ }^{4}$ J. Balajthy, ${ }^{16}$ S. Bedikian, ${ }^{19}$ E. Bernard, ${ }^{19}$ A. Bernstein,${ }^{6}$
} A. Bolozdynya, ${ }^{2}$ A. Bradley, ${ }^{2}$ D. Byram, ${ }^{18}$ S. B. Cahn, ${ }^{19}$ M. C. Carmona-Benitez, ${ }^{2,14}$ C. Chan, ${ }^{1}$ J. J. Chapman, ${ }^{1}$ A. A. Chiller, ${ }^{18}$ C. Chiller, ${ }^{18}$ K. Clark, ${ }^{2}$ T. Coffey, ${ }^{2}$ A. Currie, ${ }^{4}$ A. Curioni, ${ }^{19}$ S. Dazeley, ${ }^{6}$ L. de Viveiros, ${ }^{7}$ A. Dobi, ${ }^{16}$ J. Dobson, ${ }^{15}$ E. M. Dragowsky, ${ }^{2}$ E. Druszkiewicz, ${ }^{17}$ B. Edwards, ${ }^{19, \dagger}$ C. H. Faham, ${ }^{1,5}$ S. Fiorucci, ${ }^{1}$ C. Flores, ${ }^{13}$ R. J. Gaitskell, ${ }^{1}$ V. M. Gehman, ${ }^{5}$ C. Ghag, ${ }_{11}^{11}$ K. R. Gibson, ${ }^{2}$ M. G. D. Gilchriese, ${ }^{5}$ C. Hall, ${ }^{16}$ M. Hanhardt,,${ }^{8,9}$ S. A. Hertel, ${ }^{19}$ M. Horn, ${ }^{19}$ D. Q. Huang, ${ }^{1}$ M. Ihm, ${ }^{12}$ R. G. Jacobsen, ${ }^{12}$ L. Kastens, ${ }^{19}$ K. Kazkaz,${ }^{6}$ R. Knoche, ${ }^{16}$ S. Kyre, ${ }^{14}$ R. Lander, ${ }^{13}$ N. A. Larsen, ${ }^{19}$ C. Lee, ${ }^{2}$ D. S. Leonard, ${ }^{16}$ K. T. Lesko, ${ }^{5}$ A. Lindote, ${ }^{7}$ M. I. Lopes, ${ }^{7}$ A. Lyashenko, ${ }^{19}$ D. C. Malling, ${ }^{1}$ R. Mannino, ${ }^{10}$

D. N. McKinsey, ${ }^{19}$ D.-M. Mei, ${ }^{18}$ J. Mock, ${ }^{13}$ M. Moongweluwan, ${ }^{17}$ J. Morad, ${ }^{13}$ M. Morii, ${ }^{3}$ A. St. J. Murphy, ${ }^{15}$ C. Nehrkorn, ${ }^{14}$ H. Nelson,${ }^{14}$ F. Neves, ${ }^{7}$ J. A. Nikkel,${ }^{19}$ R. A. Ott,${ }^{13}$ M. Pangilinan, ${ }^{1}$ P. D. Parker, ${ }^{19}$ E. K. Pease,${ }^{19}$ K. Pech, ${ }^{2}$ P. Phelps, ${ }^{2}$ L. Reichhart, ${ }^{11}$ T. Shutt, ${ }^{2}$ C. Silva, ${ }^{7}$ W. Skulski, ${ }^{17}$ C. J. Sofka, ${ }^{10}$ V. N. Solovov, ${ }^{7}$ P. Sorensen, ${ }^{6}$ T. Stiegler, ${ }^{10}$ K. O'Sullivan, ${ }^{19}$ T. J. Sumner, ${ }^{4}$ R. Svoboda, ${ }^{13}$ M. Sweany, ${ }^{13}$ M. Szydagis, ${ }^{13}$ D. Taylor, ${ }^{9}$ B. Tennyson, ${ }^{19}$ D. R. Tiedt, ${ }^{8}$ M. Tripathi, ${ }^{13}$ S. Uvarov, ${ }^{13}$ J. R. Verbus,${ }^{1}$ N. Walsh, ${ }^{13}$ R. Webb ${ }^{10}$ J. T. White, ${ }^{10,}{ }^{*}$ D. White,${ }^{14}$ M. S. Witherell, ${ }^{14}$ M. Wlasenko, ${ }^{3}$ F. L. H. Wolfs, ${ }^{17}$ M. Woods, ${ }^{13}$ and C. Zhang ${ }^{18}$

(LUX Collaboration)

\author{
${ }^{1}$ Department of Physics, Brown University, 182 Hope Street, Providence, Rhode Island 02912, USA \\ ${ }^{2}$ Department of Physics, Case Western Reserve University, 10900 Euclid Avenue, Cleveland, Ohio 44106, USA \\ ${ }^{3}$ Department of Physics, Harvard University, 17 Oxford Street, Cambridge, Massachusetts 02138, USA \\ ${ }^{4}$ High Energy Physics, Imperial College London, Blackett Laboratory, London SW7 2BZ, United Kingdom \\ ${ }^{5}$ Lawrence Berkeley National Laboratory, 1 Cyclotron Road, Berkeley, California 94720, USA \\ ${ }^{6}$ Lawrence Livermore National Laboratory, 7000 East Avenue, Livermore, California 94550, USA \\ ${ }^{7}$ LIP-Coimbra, Department of Physics, University of Coimbra, Rua Larga, 3004-516 Coimbra, Portugal \\ ${ }^{8}$ South Dakota School of Mines and Technology, 501 East St Joseph Street, Rapid City, South Dakota 57701, USA \\ ${ }^{9}$ South Dakota Science and Technology Authority, Sanford Underground Research Facility, Lead, South Dakota 57754, USA \\ ${ }^{10}$ Department of Physics, Texas A \& M University, College Station, Texas 77843, USA \\ ${ }^{11}$ Department of Physics and Astronomy, University College London, Gower Street, London WC1E 6BT, United Kingdom \\ ${ }^{12}$ Department of Physics, University of California Berkeley, Berkeley, California 94720, USA \\ ${ }^{13}$ Department of Physics, University of California Davis, One Shields Avenue, Davis, California 95616, USA \\ ${ }^{14}$ Department of Physics, University of California Santa Barbara, Santa Barbara, California 93106, USA \\ ${ }^{15}$ SUPA, School of Physics and Astronomy, University of Edinburgh, Edinburgh, EH9 3JZ, United Kingdom \\ ${ }^{16}$ Department of Physics, University of Maryland, College Park, Maryland 20742, USA \\ ${ }^{17}$ Department of Physics and Astronomy, University of Rochester, Rochester, New York 14627, USA \\ ${ }^{18}$ Department of Physics, University of South Dakota, 414E Clark Street, Vermillion, South Dakota 57069, USA \\ ${ }^{19}$ Department of Physics, Yale University, 217 Prospect Street, New Haven, Connecticut 06511, USA \\ (Received 30 October 2013; revised manuscript received 21 January 2014; published 4 March 2014)
}

The Large Underground Xenon (LUX) experiment is a dual-phase xenon time-projection chamber operating at the Sanford Underground Research Facility (Lead, South Dakota). The LUX cryostat was filled for the first time in the underground laboratory in February 2013. We report results of the first WIMP search data set, taken during the period from April to August 2013, presenting the analysis of 85.3 live days of data with a fiducial volume of $118 \mathrm{~kg}$. A profile-likelihood analysis technique shows our data to be consistent with the background-only hypothesis, allowing $90 \%$ confidence limits to be set on spin-independent WIMP-nucleon elastic scattering with a minimum upper limit on the cross section of $7.6 \times 10^{-46} \mathrm{~cm}^{2}$ at a WIMP mass of $33 \mathrm{GeV} / \mathrm{c}^{2}$. We find that the LUX data are in disagreement with lowmass WIMP signal interpretations of the results from several recent direct detection experiments.

DOI: 10.1103/PhysRevLett.112.091303

PACS numbers: 95.35.+d, 29.40.Gx, 95.55.Vj

Convincing evidence for the existence of particle dark matter is derived from observations of the Universe on scales ranging from the galactic to the cosmological [1-3].
Increasingly detailed studies of the cosmic microwave background anisotropies have implied the abundance of dark matter with remarkable precision [4,5]. One favored 
class of dark matter candidates, the weakly interacting massive particle (WIMP), may be amenable to direct detection in laboratory experiments through its interactions with ordinary matter $[6,7]$. The WIMPs that constitute our galactic halo would scatter elastically with nuclei, generating recoil energies of several $\mathrm{keV}$.

We report here the first results from the Large Underground Xenon (LUX) experiment, currently operating 4850 feet below ground (4300 m w.e.) at the Sanford Underground Research Facility (SURF) [8,9] in Lead, South Dakota. Fluxes of cosmic-ray muons, neutrons, and $\gamma$ rays at SURF have been published elsewhere [10]. Inside the cavern, a $7.6 \mathrm{~m}$ diameter by $6.1 \mathrm{~m}$ tall cylindrical water tank provides shielding to the detector. These large reductions in external radiation provide the lowbackground environment required for the rare event search.

The LUX detector holds $370 \mathrm{~kg}$ of liquid xenon, with $250 \mathrm{~kg}$ actively monitored in a dual-phase (liquid-gas) time-projection chamber (TPC) measuring $47 \mathrm{~cm}$ in diameter and $48 \mathrm{~cm}$ in height (cathode to gate) [11]. Interactions in the liquid produce prompt scintillation (S1) and ionization electrons that drift in an applied electric field [12]. Electrons are extracted into the gas, where they produce electroluminescence $(S 2)$. $S 1$ and $S 2$ signals are used to reconstruct the deposited energy and their ratio is used to discriminate nuclear recoils (NR) from electron recoils (ER). Light signals are detected via two arrays of 61 photomultiplier tubes (PMTs), one array above the active region in the gas and one below it in the liquid [13]. During this search, three PMTs were left unbiased, two in the top array and one in the bottom (one PMT was grounded and the others produced an abnormal increase in event rate). The $(x, y)$ position of an interaction is determined from localization of the $S 2$ signal in the top PMT array, with the difference in time between the $S 1$ and $S 2$ representing event depth. The $(x, y)$ position resolution for small $S 2$ signals (such as those in the WIMP search region in terms of both energy and fiducial volume) is $4-6 \mathrm{~mm}$, and even better at higher energies. $S 2$ pulse areas measured from the bottom PMT array alone $\left(S 2_{b}\right)$ are used in later analysis, avoiding events leaking into the signal region due to uncollected $S 2$ light from the deactivated PMTs in the top array.

Throughout the WIMP search, the xenon vessel was thermally isolated with an outer vacuum vessel providing thermal stability of $\Delta T<0.2 \mathrm{~K}$, pressure stability $\Delta P / P<1 \%$, and liquid level variation of $<0.2 \mathrm{~mm}$ [14] (measured from stability of $S 2$ width). An electric field of $181 \mathrm{~V} / \mathrm{cm}$ was applied across the WIMP target region providing a measured average electron drift velocity of $1.51 \pm 0.01 \mathrm{~mm} / \mu \mathrm{s}$. Above the drift region, a field of $6.0 \mathrm{kV} / \mathrm{cm}$ is applied to the gas $(3.1 \mathrm{kV} / \mathrm{cm}$ in the liquid), producing a best-fit electron extraction efficiency of $0.65 \pm 0.01$. The distribution of the number of $S 2$ photoelectrons observed for each extracted electron has a mean of 24.6 and an rms variation of 7.0. If only $S 2_{b}$ is considered, the mean is 10.4 and the rms is 4.5 .

Purification of the xenon, circulating through a hotzirconium getter $(229 \mathrm{~kg} /$ day $)$, resulted in mean electron drift-lengths, before capture by electronegative impurities, between $87 \pm 9$ and $134 \pm 15 \mathrm{~cm}$ during WIMP search.

The data acquisition (DAQ) threshold is set such that $\gtrsim 95 \%$ of all single photoelectron (phe) pulses in each PMT are recorded to disk $[15,16]$. A digital trigger identifies events for further analysis, with nonadjacent PMTs grouped together into 16 trigger channels. The trigger requires that at least two of these channels have greater than 8 phe within a $2 \mu$ s window, with a trigger efficiency $>99 \%$ for $S 2$ signals above the analysis threshold of 200 phe. Every pulse of light digitized by the DAQ within $\pm 500 \mu$ s of the trigger time (324 $\mu$ s maximum drift time) was allocated to a triggered event for further analysis, ensuring that corresponding $S 1$ and $S 2$ pulses can always be associated. Additionally, data between triggered events are retained to verify that the detector is quiet in the period leading up to, and following, the events.

This initial dark matter search consists of 85.3 live days of WIMP search data acquired between April 21 and August 8, 2013. The live-time calculation accounts for the DAQ dead time $(0.2 \%)$, a $1-4 \mathrm{~ms}$ trigger hold-off to prevent additional triggers following large $S 2$ pulses $(2.2 \%)$, and exclusions for periods of detector instability $(0.8 \%)$.

A nonblind analysis was conducted on the 85.3 live days of WIMP search data, where only a minimal set of data quality cuts, with high acceptance, was employed to reduce the scope for bias. The low total event rate in the center of the detector minimizes the rate of misidentified ER background events. For this initial analysis of the first low-background operation of the instrument, both the calibration and WIMP search data were used to understand and develop analysis algorithms.

Waveforms from each PMT are summed across all channels and then searched with pulse finding algorithms to select viable signals. The identification of an $S 1$ signal requires at least two PMTs to detect more than 0.25 phe each within $100 \mathrm{~ns}$ of each other. The average dark count rate for each PMT in the array is $12 \mathrm{~Hz}$. An estimate of the rate of events where an accidental 2 phe dark count coincidence fakes an $S 1$ preceding a valid $S 2$-only event in the NR signal region is $1.2 \mathrm{nHz}(0.009$ events in the search dataset) [16].

Events containing exactly one $S 1$ within the maximum drift time (324 $\mu$ s) preceding a single $S 2$, representative of expected elastic scattering of WIMPs, are selected for further analysis. Additionally, we require a raw $S 2$ pulse size greater than 200 phe ( $\sim 8$ extracted electrons). This excludes a small number of single-extracted-electron-type events (having poor event reconstruction) and those from the detector walls with small $S 2$ signals (having poorly 
reconstructed positions). The 200 phe threshold for $S 2$ light was optimized by studying the efficiency of the reconstruction algorithms with the calibration data and by observing the background outside the WIMP search energy range. Single scatter ER and NR acceptance was measured with dedicated tritium $\left(\beta^{-}\right)$, AmBe, and ${ }^{252} \mathrm{Cf}$ (neutron) datasets. Simulated NR event waveforms, generated with LUXSIM [17,18], were analyzed with the complete data processing framework to validate the analysis efficiencies measured with data. $S 2$ finding efficiency is $>99 \%$ above the analysis threshold of 200 phe. The relative efficiency for NR detection is dominated by the $S 1$ identification (shown in Fig. 1). Absolute efficiency is estimated through visual inspection of waveforms from NR calibration data to be $98 \%$, which is in agreement with the value measured by an injection of tritiated methane of
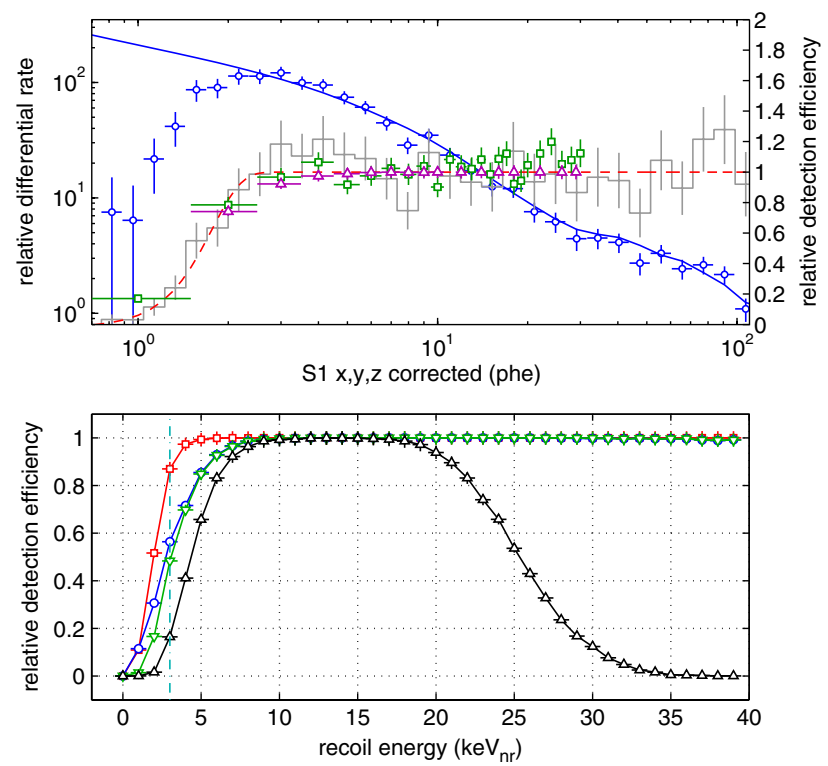

FIG. 1 (color online). Top: Comparison of AmBe data (blue circles) with NEST simulations (blue line), showing excellent agreement above the 2 phe threshold (left axis). The gray histogram and fitted dashed red line show the relative efficiency for detection of nuclear recoils from AmBe data (right axis). Overlaid are the ER detection efficiency from tritium data (green squares), applied to the ER background model in the profile likelihood analysis, and the efficiency from full detector NR simulations treated as real data in terms of the digitized MC-truth $S 1$ phe (purple triangles), applied to the WIMP signal model. The efficiency calculation here does not include $S 1$ or $S 2$ area thresholds. Bottom: WIMP detection efficiency as a function of nuclear recoil energy for events with a corrected $S 1$ between 2 and 30 phe and a $S 2$ signal greater than 200 phe (black upward triangle), the efficiency used directly in the profile likelihood analysis. The efficiency for individually detecting an $S 2$ (red square) or $S 1$ (blue circle) signal (without the application of any analysis thresholds) are also shown, along with that after the single scatter requirement (green downward triangle). The cyan dashed line indicates the threshold in keVnr below which we assume no light or charge response in the PLR calculation. known activity. All cuts and efficiencies combine to give an overall WIMP detection efficiency of $50 \%$ at $4.3 \mathrm{keV}_{\mathrm{nr}}$ $\left(17 \%\right.$ at $3 \mathrm{keV}_{\mathrm{nr}}$ and $>95 \%$ above $\left.7.5 \mathrm{keV}_{\mathrm{nr}}\right)$, shown in Fig. 1.

A radial fiducial cut was placed at $18 \mathrm{~cm}$ (Fig. 2), defined by the positions of decay products from $\mathrm{Rn}$ daughters implanted on the detector walls. This population, primarily sub-NR band but intersecting the signal region at the lowest energies, is visible (along with other expected backgrounds) on the detector walls in Fig. 2. This cut was chosen by selecting those sub-NR band events outside of the WIMP search energy range ( $S 1>30$ phe). In height, the fiducial volume was defined by a drift time between 38 and $305 \mu$ s to reduce backgrounds from the PMT arrays and electrodes. This cut was chosen by examining the event rate as a function of depth outside of the WIMP search energy range $(S 1>30$ phe) and confirmed with Monte Carlo simulations. The fiducial target mass is calculated to be $118.3 \pm 6.5 \mathrm{~kg}$ from assessment of the homogeneous tritium data, and confirmed from assessment of the homogeneous ${ }^{83 m} \mathrm{Kr}$ data, whose mono-energetic peak provides excellent tagging to monitor dispersal of the ${ }^{83 m} \mathrm{Kr}$ throughout the detector volume.

Periods of live-time with high rates of single electron backgrounds ( $\gtrsim 4$ extracted electrons per $1 \mathrm{~ms}$ event window) are removed [19-21]. The associated loss of live-time is $0.8 \%$ (measured from assessment of the full dataset, including nontriggered regions), primarily removing periods following large $S 2$ pulses.

Extensive calibrations were acquired with internal ER sources (tritiated methane, ${ }^{83 m} \mathrm{Kr}$ ) and NR calibrations were performed with external neutron sources (AmBe, ${ }^{252} \mathrm{Cf}$ ). The ER sources were injected into the xenon gas system and allowed to disperse uniformly, achieving a homogeneous calibration of the active region. In particular, we developed a novel tritiated-methane $\beta^{-}$source $\left(E_{\max } \simeq 18 \mathrm{keV}\right)$ that produces events extending below

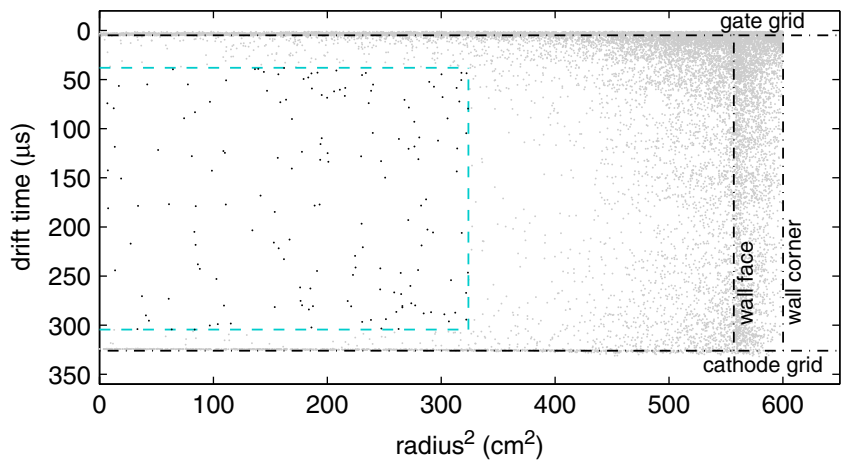

FIG. 2 (color online). Spatial distribution of all events with position-corrected $S 1$ in the range $2-30$ phe from the 85.3 live days of WIMP search data. The cyan dashed line indicates the fiducial volume. The physical locations of the cathode and gate grids and the detector walls (where the vertical PTFE walls of the TPC form a dodecagon) are also shown. 


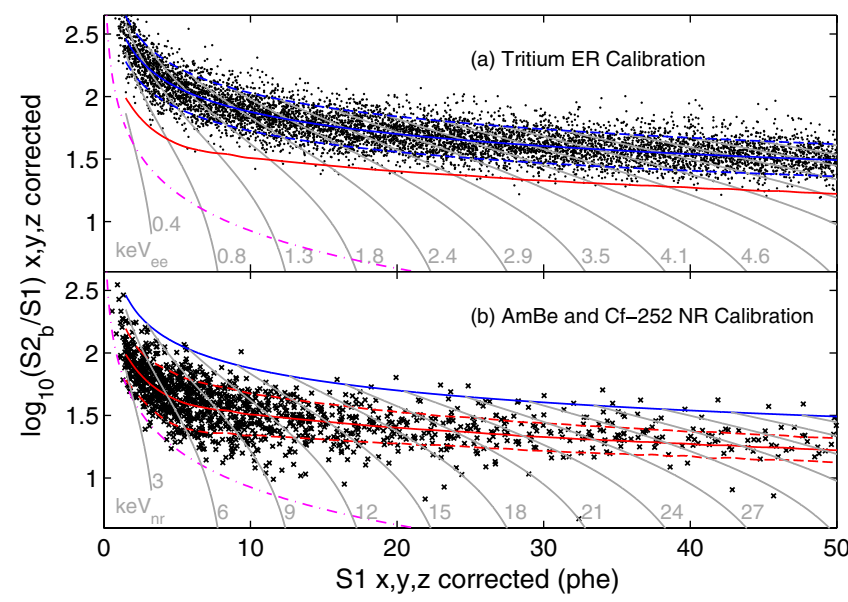

FIG. 3 (color online). Calibrations of detector response in the $118 \mathrm{~kg}$ fiducial volume. The ER (tritium, panel (a) and NR (AmBe and ${ }^{252} \mathrm{Cf}$, panel (b)) calibrations are depicted, with the means (solid line) and $\pm 1.28 \sigma$ contours (dashed line). This choice of band width (indicating $10 \%$ band tails) is for presentation only. Panel (a) shows fits to the high statistics tritium data, with fits to simulated NR data shown in panel (b), representing the parameterizations taken forward to the profile likelihood analysis. The ER plot also shows the NR band mean and vice versa. Gray contours indicate constant energies using an $S 1-S 2$ combined energy scale (same contours on each plot). The dotdashed magenta line delineates the approximate location of the minimum S2 cut.

$1 \mathrm{keV}_{\text {ee }}$, allowing ER band (Fig. 3) and detection efficiency calibrations (Fig. 1) with unprecedented accuracy; the tritiated methane is subsequently fully removed by circulating the xenon through the getter.

A ${ }^{83 m} \mathrm{Kr}$ injection was performed weekly to determine the free electron lifetime and the three-dimensional correction functions for photon detection efficiency, which combine the effects of geometric light collection and PMT quantum efficiency (corrected $S 1$ and $S 2$ ). The 9.4 and $32.1 \mathrm{keV}$ depositions [22] demonstrated the stability of the $S 1$ and $S 2$ signals in time, the latter confirmed with measurements of the single extracted electron response. ${ }^{131 m} \mathrm{Xe}$ and ${ }^{129 m} \mathrm{Xe}$ (164 and $236 \mathrm{keV}$ deexcitations) afforded another internal calibration, providing a crosscheck of the photon detection and electron extraction efficiencies. To model these efficiencies, we employed field- and energy-dependent absolute scintillation and ionization yields from NEST [23-25], which provides an underlying physics model, not extrapolations, where only detector parameters such as photon detection efficiency, electron extraction efficiency and single electron response are inputs to the simulation. Using a Gaussian fit to the single phe area [26], together with the $S 1$ spectrum of tritium events, the mean $S 1$ photon detection efficiency was determined to be $0.14 \pm 0.01$, varying between 0.11 and 0.17 from the top to the bottom of the active region. This is estimated to correspond to $8.8 \mathrm{phe} / \mathrm{keV}_{\text {ee }}$ (electron-

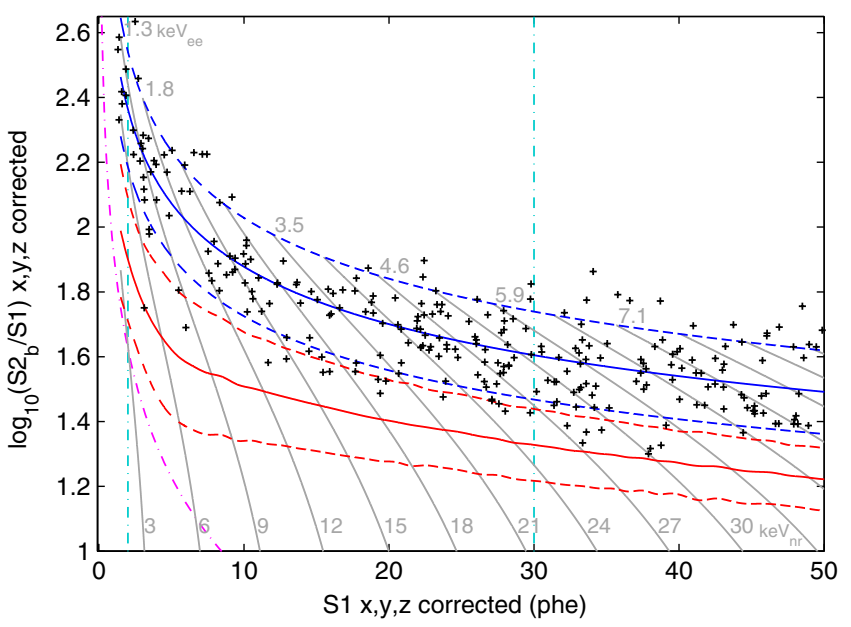

FIG. 4 (color online). The LUX WIMP signal region. Events in the $118 \mathrm{~kg}$ fiducial volume during the 85.3 live-day exposure are shown. Lines as shown in Fig. 3, with vertical dashed cyan lines showing the 2-30 phe range used for the signal estimation analysis.

equivalent energy) for $122 \mathrm{keV} \gamma$ rays at zero field [23]. This high photon detection efficiency (unprecedented in a xenon WIMP-search TPC) is responsible for the low threshold and good discrimination observed [27].

Detector response to ER and NR calibration sources is presented in Fig. 3. Comparison of $\mathrm{AmBe}$ data with simulation permits extraction of NR detection efficiency (Fig. 1), which is in excellent agreement with that obtained using other data sets $\left({ }^{252} \mathrm{Cf}\right.$ and tritium). We describe the populations as a function of $S 1$ (Figs. 3 and 4), as this provides the dominant component of detector efficiency. We also show contours of approximated constant-energy [28], calculated from a linear combination of $S 1$ and $S 2$ $[24,27,29]$ generated by converting the measured pulse areas into original photons and electrons (given their efficiencies).

A parameterization (for $S 2$ at a given $S 1$ ) of the ER band from the high-statistics tritium calibration is used to characterize the background. In turn, the NR calibration is more challenging, partly due to the excellent self-shielding of the detector. Neutron calibrations therefore include systematic effects not applicable to the WIMP signal model, such as multiple-scattering events (including those where scatters occur in regions of differing field) or coincident Compton scatters from $\mathrm{AmBe}$ and ${ }^{252} \mathrm{Cf} \gamma$ rays and (n, $\left.\gamma\right)$ reactions. These effects produce the dispersion observed in data, which is well modeled in our simulations (in both band mean and width, verifying the simulated energy resolution), and larger than that expected from WIMP scattering. Consequently, these data cannot be used directly to model a signal distribution. For different WIMP masses, simulated $S 1$ and $S 2$ distributions are obtained, accounting for their unique energy spectra.

The ratio of $\mathrm{keV}_{\mathrm{ee}}$ to nuclear recoil energy $\left(\mathrm{keV}_{\mathrm{nr}}\right)$ relies on both $S 1$ and $S 2$, using the conservative technique presented in [29] (Lindhard with $k=0.110$, compared to the default Lindhard value of 0.166 and the implied best-fit 
value of 0.135 from [29]). NR data are consistent with an energy-dependent, nonmonotonic reduced light yield with respect to zero field [30] with a minimum of 0.77 and a maximum of 0.82 in the range $3-25 \mathrm{keV}_{\mathrm{nr}}$ [23] (compared with $0.90-0.95$ used by previous xenon experiments for significantly higher electric fields [47,50]). This is understood to stem from additional, anticorrelated portioning into the ionization channel.

The observed ER background in the range $0.9-5.3 \mathrm{keV}_{\text {ee }}$ within the fiducial volume was $3.6 \pm 0.3 \mathrm{mDRU}_{\mathrm{ee}}$ averaged over the WIMP search dataset (summarized in Table I). Backgrounds from detector components were controlled through a material screening program at the Soudan LowBackground Counting Facility (SOLO) and the LBNL lowbackground counting facility [13,26,31]. Krypton as a mass fraction of xenon was reduced from $130 \mathrm{ppb}$ in the purchased xenon to 4 ppt using gas charcoal chromatography [32].

Radiogenic backgrounds were extensively modeled using LUXSIM, with approximately $73 \%$ of the low-energy $\gamma$-ray background originating from the materials in the R8778 PMTs and the rest from other construction materials. This demonstrated consistency between the observed $\gamma$-ray energy spectra and position distribution [33], and the expectations based on the screening results and the independent assay of the natural $\mathrm{Kr}$ concentration of $3.5 \pm$ $1 \mathrm{ppt}(\mathrm{g} / \mathrm{g})$ in the xenon gas [34] where we assume an isotopic abundance of ${ }^{85} \mathrm{Kr} /{ }^{\text {nat }} \mathrm{Kr} \sim 2 \times 10^{-11} \quad[33,35]$. Isotopes created through cosmogenic production were also considered, including measured levels of ${ }^{60} \mathrm{Co}$ in $\mathrm{Cu}$ components. In situ measurements determined additional intrinsic background levels in xenon from ${ }^{214} \mathrm{~Pb}$ (from the ${ }^{222} \mathrm{Rn}$ decay chain) [36], and cosmogenically-produced ${ }^{127} \mathrm{Xe}\left(T_{1 / 2}=36.4\right.$ days $),{ }^{129 m} \mathrm{Xe}\left(T_{1 / 2}=8.9\right.$ days $)$, and ${ }^{131 m} \mathrm{Xe}\left(T_{1 / 2}=11.9\right.$ days). The rate from ${ }^{127} \mathrm{Xe}$ in the WIMP search energy window is estimated to decay from $0.87 \mathrm{mDRU}_{\text {ee }}$ at the start of the WIMP search dataset to $0.28 \mathrm{mDRU}_{\mathrm{ee}}$ at the end, with late-time background measurements being consistent with those originating primarily from the long-lived radioisotopes.

TABLE I. Predicted background rates in the fiducial volume $\left(0.9-5.3 \mathrm{keV}_{\mathrm{ee}}\right)$ [33]. We show contributions from the $\gamma$ rays of detector components (including those cosmogenically activated), the time-weighted contribution of activated xenon, ${ }^{222} \mathrm{Rn}$ (best estimate 0.2 mDRUee from ${ }^{222} \mathrm{Rn}$ chain measurements) and ${ }^{85} \mathrm{Kr}$. The errors shown are both from simulation statistics and those derived from the rate measurements of time-dependent backgrounds. $1 \mathrm{mDRU}_{\mathrm{ee}}$ is $10^{-3}$ events $/ \mathrm{keV}_{\mathrm{ee}} / \mathrm{kg} /$ day.

\begin{tabular}{lc}
\hline \hline Source & Background rate, $\mathrm{mDRU}_{\mathrm{ee}}$ \\
\hline$\gamma$ rays & $1.8 \pm 0.2_{\text {stat }} \pm 0.3_{\text {sys }}$ \\
${ }^{127} \mathrm{Xe}$ & $0.5 \pm 0.02_{\text {stat }} \pm 0.1_{\text {sys }}$ \\
${ }^{214} \mathrm{~Pb}$ & $0.11-0.22(90 \%$ C.L. $)$ \\
${ }^{85} \mathrm{Kr}$ & $0.13 \pm 0.07_{\text {sys }}$ \\
Total predicted & $2.6 \pm 0.2_{\text {stat }} \pm 0.4_{\text {sys }}$ \\
Total observed & $3.6 \pm 0.3_{\text {stat }}$ \\
\hline \hline
\end{tabular}

The neutron background in LUX is predicted from detailed detector BG simulations to produce 0.06 single scatters with $S 1$ between 2 and 30 phe in the 85.3 live-day data set. This was considered too low to include in the PLR. The value was constrained by multiple-scatter analysis in the data, with a conservative $90 \%$ upper C.L. placed on the number of expected neutron single scatters of 0.37 events.

We observed 160 events between 2 and 30 phe $(S 1)$ within the fiducial volume in 85.3 live days of search data (shown in Fig. 4), with all observed events being consistent with the predicted background of electron recoils. The average discrimination (with $50 \%$ NR acceptance) for $S 1$ from $2-30$ phe is $99.6 \pm 0.1 \%$; hence, $0.64 \pm 0.16$ events from ER leakage are expected below the NR mean, for the search dataset. The spatial distribution of the events matches that expected from the ER backgrounds in full detector simulations. We select the upper bound of 30 phe $(S 1)$ for the signal estimation analysis to avoid additional background from the $5 \mathrm{keV}_{\mathrm{ee}} \mathrm{x}$ ray from ${ }^{127} \mathrm{Xe}$.

Confidence intervals on the spin-independent WIMPnucleon cross section are set using a profile likelihood ratio (PLR) test statistic [37], exploiting the separation of signal and background distributions in four physical quantities: radius, depth, light $(S 1)$, and charge $(S 2)$. The fit is made over the parameter of interest plus three Gaussianconstrained nuisance parameters which encode uncertainty in the rates of ${ }^{127} \mathrm{Xe}, \gamma$ rays from internal components and the combination of ${ }^{214} \mathrm{~Pb}$ and ${ }^{85} \mathrm{Kr}$. The distributions, in the observed quantities, of the four model components are as described above and do not vary in the fit: with the nonuniform spatial distributions of $\gamma$-ray backgrounds and $\mathrm{x}$-ray lines from ${ }^{127} \mathrm{Xe}$ obtained from energydeposition simulations [33]. The PLR operates within the fiducial region but the spatial background models were validated using data from outside the fiducial volume.

The energy spectrum of WIMP-nucleus recoils is modeled using a standard isothermal Maxwellian velocity distribution [38], with $v_{0}=220 \mathrm{~km} / \mathrm{s} ; v_{\mathrm{esc}}=544 \mathrm{~km} / \mathrm{s}$; $\rho_{0}=0.3 \mathrm{GeV} / \mathrm{cm}^{3}$; average Earth velocity of $245 \mathrm{~km} \mathrm{~s}^{-1}$, and Helm form factor $[39,40]$. We conservatively model no signal below $3.0 \mathrm{keV}_{\mathrm{nr}}$ (the lowest energy for which a direct light yield measurement exists [30,41], whereas indirect evidence of charge yield exists down to $1 \mathrm{keV}_{\mathrm{nr}}$ [42]). We do not profile the uncertainties in NR yield, assuming a model which provides excellent agreement with LUX data (Fig. 1 and [43]), in addition to being conservative compared to past works [23]. We also do not account for uncertainties in astrophysical parameters, which are beyond the scope of this work (but are discussed in [44]). Signal models in $S 1$ and $S 2$ are obtained for each WIMP mass from full simulations.

The observed PLR for zero signal is entirely consistent with its simulated distribution, giving a $p$ value for the background-only hypothesis of 0.35 . The $90 \%$ C.L. upper limit on the number of expected signal events ranges, over 


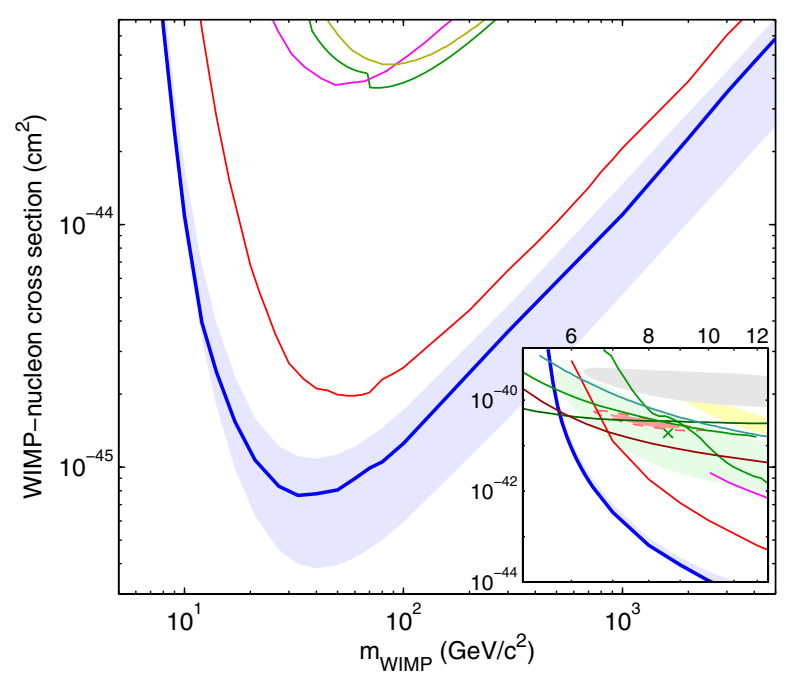

FIG. 5 (color online). The LUX 90\% confidence limit on the spin-independent elastic WIMP-nucleon cross section (blue), together with the $\pm 1 \sigma$ variation from repeated trials, where trials fluctuating below the expected number of events for zero BG are forced to 2.3 (blue shaded). We also show Edelweiss II [45] (dark yellow line), CDMS II [46] (green line), ZEPLIN-III [47] (magenta line), CDMSlite [48] (dark green line), XENON10 S2-only [20] (brown line), SIMPLE [49] (light blue line), and XENON100 225 live-day [50] (red line) results. The inset (same axis units) also shows the regions measured from annual modulation in CoGeNT [51] (light red, shaded), along with exclusion limits from low threshold re-analysis of CDMS II data [52] (upper green line), 95\% allowed region from CDMS II silicon detectors [53] (green shaded) and centroid (green x), 90\% allowed region from CRESST II [54] (yellow shaded) and DAMA/LIBRA allowed region [55] interpreted by [56] (grey shaded). (results sourced from DMTools [57]).

WIMP masses, from 2.4 to 5.3. A variation of one standard deviation in detection efficiency shifts the limit by an average of only $5 \%$. The systematic uncertainty in the position of the NR band was estimated by averaging the difference between the centroids of simulated and observed AmBe data in $\log \left(S 2_{b} / S 1\right)$. This yielded an uncertainty of 0.044 in the centroid, which propagates to a maximum uncertainty of $25 \%$ in the high mass limit.

The $90 \%$ upper C.L. cross sections for spin-independent WIMP models are thus shown in Fig. 5 with a minimum cross section of $7.6 \times 10^{-46} \mathrm{~cm}^{2}$ for a WIMP mass of $33 \mathrm{GeV} / \mathrm{c}^{2}$. This represents a significant improvement over the sensitivities of earlier searches $[46,47,50,51]$. The low energy threshold of LUX permits direct testing of low mass WIMP hypotheses where there are potential hints of signal [46,51,54,55]. These results do not support such hypotheses based on spin-independent isospin-invariant WIMP-nucleon couplings and conventional astrophysical assumptions for the WIMP halo, even when using a conservative interpretation of the existing low-energy nuclear recoil calibration data for xenon detectors.
LUX will continue operations at SURF during 2014 and 2015. Further engineering and calibration studies will establish the optimal parameters for detector operations, with potential improvements in applied electric fields, increased calibration statistics, decaying backgrounds and an instrumented water tank veto further enhancing the sensitivity of the experiment. Subsequently, we will complete the ultimate goal of conducting a blinded 300 live-day WIMP search further improving sensitivity to explore significant new regions of WIMP parameter space.

This work was partially supported by the U.S. Department of Energy (DOE) under Awards No. DEFG02-08ER41549, No. DE-FG02-91ER40688, No. DEFG02-95ER40917, No. DE-FG02-91ER40674, No. DE-NA0000979, No. DE-FG02-11ER41738, No. DESC0006605, No. DE-AC02-05CH11231, No. DE-AC5207NA27344, and No. DE-FG01-91ER40618; the U.S. National Science Foundation under Awards No. PHYS-0750671, No. PHY-0801536, No. PHY-1004661, No. PHY-1102470, No. PHY-1003660, No. PHY-1312561, No. PHY-1347449; the Research Corporation Grant No. RA0350; the Center for Ultra-low Background Experiments in the Dakotas (CUBED); and the South Dakota School of Mines and Technology (SDSMT). LIP-Coimbra acknowledges funding from Fundação para a Ciência e Tecnologia (FCT) through the Project-Grant No. CERN/FP/123610/2011. Imperial College and Brown University thank the UK Royal Society for travel funds under the International Exchange Scheme (IE120804). The UK groups acknowledge institutional support from Imperial College London, University College London, and Edinburgh University, and from the Science \& Technology Facilities Council for $\mathrm{Ph}$. D. studentship No. ST/K502042/1 (AB). The University of Edinburgh is a charitable body, registered in Scotland, with registration No. SC005336. This research was conducted using computational resources and services at the Center for Computation and Visualization, Brown University. We acknowledge the work of the following engineers who played important roles during the design, construction, commissioning, and operation phases of LUX: S. Dardin from Berkeley, B. Holbrook, R. Gerhard, and J. Thomson from University of California, Davis; and G. Mok, J. Bauer, and D. Carr from Lawrence Livermore National Laboratory. We gratefully acknowledge the logistical and technical support and the access to laboratory infrastructure provided to us by the Sanford Underground Research Facility (SURF) and its personnel at Lead, South Dakota. SURF was developed by the South Dakota Science and Technology authority, with an important philanthropic donation from T. Denny Sanford, and is operated by Lawrence Berkeley National Laboratory for the Department of Energy, Office of High Energy Physics. 
*Deceased.

Corresponding author.

blair.edwards@yale.edu

[1] G. R. Blumenthal, S. Faber, J. R. Primack, and M. J. Rees, Nature (London) 311, 517 (1984).

[2] M. Davis, G. Efstathiou, C. S. Frenk and S. D. M. White, Astrophys. J. 292, 371 (1985).

[3] D. Clowe, M. Bradac, A. H. Gonzalez, M. Markevitch, S. W. Randell, C. Jones and D. Zaritsky, Astrophys. J. 648, L109 (2006).

[4] C. L. Bennett et al., Astrophys. J. Suppl. Ser. 208 (2013) 20.

[5] P. Ade et al. (Planck Collaboration), arXiv:1303.5076.

[6] M. W. Goodman and E. Witten, Phys. Rev. D 31, 3059 (1985).

[7] J. L. Feng, Annu. Rev. Astron. Astrophys. 48, 495 (2010).

[8] K. T. Lesko et al., arXiv:1108.0959.

[9] K. T. Lesko, Eur. Phys. J. Plus 127, 107 (2012).

[10] D.-M. Mei, C. Zhang, K. Thomas and F. Gray, Astropart. Phys. 34, 33 (2010); F. E. Gray, C. Ruybal, J. Totushek, D.-M. Mei, K. Thomas, C. Zhang, Nucl. Instrum. Methods A 638, 63 (2011).

[11] D. S. Akerib et al. (LUX Collaboration), Nucl. Instrum. Methods A 704, 111 (2013).

[12] V. Chepel, H. Araujo, JINST 8, R04001 (2013).

[13] D. S. Akerib et al. (LUX Collaboration), Nucl. Instrum. Methods A 703, 1 (2013).

[14] P. Phelps, Ph.D thesis, Case Western Reserve University, 2014 (to be published).

[15] D. S. Akerib et al. (LUX Collaboration), Nucl. Instrum. Methods Phys. Res., Sect. A 668, 1 (2012).

[16] J. J. Chapman, Ph.D thesis, Brown University, 2014 (to be published)

[17] D. S. Akerib et al. (LUX Collaboration), Nucl. Instrum. Methods A 675, 63 (2012).

[18] J. Mock et al., arXiv:1310.1117.

[19] B. Edwards et al., Astropart. Phys., 30, 54 (2008).

[20] J. Angle et al., Phys. Rev. Lett. 107, 051301 (2011).

[21] E. Santos et al., J. High Energy Phys. 12 (2011) 115.

[22] L. W. Kastens, S. B. Cahn, A. Manzur, D. N. McKinsey, Phys. Rev. C 80, 045809 (2009); A. Manalaysay et al., Rev. Sci. Instrum. 81, 073303 (2010).

[23] M. Szydagis, A. Fyhrie, D. Thorngren, and M. Tripathi, JINST 8, C10003 (2013).

[24] M. Szydagis, N. Barry, K. Kazkaz, J. Mock, D. Stolp, M. Sweany, M. Tripathi, S. Uvarov, N. Walsh, and M. Woods, JINST 6, P10002 (2011).

[25] L. Baudis, H. Dujmovic, C. Geis, A. James, A. Kish, A. Manalaysay, T. Marrodán Undagoitia, and M. Schumann, Phys. Rev. D 87, 115015 (2013); E. Aprile, et al., Phys. Rev. D 86, 112004 (2012).

[26] C. H. Faham, Ph.D thesis, Brown University, 2013.
[27] C. E. Dahl, Ph. D. thesis, Princeton University, 2009.

[28] P. Sorensen, Phys. Rev. D 86, 101301 (2012).

[29] P. Sorensen and C. E. Dahl, Phys. Rev. D 83, 063501 (2011).

[30] G. Plante, E. Aprile, R. Budnik, B. Choi, K.-L. Giboni, L. W. Goetzke, R. F. Lang, K. E. Lim, and A. J. Melgarejo Fernandez, Phys. Rev. C 84, 045805 (2011).

[31] D. S. Akerib et al. (LUX Collaboration), arXiv:1112.1376.

[32] A. I. Bolozdynya, P. P. Brusov, T. Shutt, C. E. Dahl, and J. Kwong, Nucl. Instrum. Methods Phys. Res., Sect. A 579, 50 (2007); D. S. Akerib et al. (LUX Collaboration) (to be published).

[33] LUX Backgrounds Paper (to be published).

[34] A. Dobi, C. Davis, C. Hall, T. Langford, S. Slutsky, and Y.-R. Yen, Nucl. Instrum. Methods Phys. Res., Sect. A 665, 1 (2011).

[35] C. Lee (LUX Collaboration), LUX $\mathrm{Kr}$ removal system, APS March meeting 2013, http://luxdarkmatter.org/talks/ APSApr2013.pdf.

[36] K. Gibson (LUX Collaboration), The LUX Experiment TAUP 2013, https://conferences.lbl.gov/contributionDisplay .py? sessionId $=19 \&$ contribId $=181 \&$ confId $=36$.

[37] G. Cowan, K. Cranmer, E. Gross, and O. Vitells, Eur. Phys. J. C 71, 1554 (2011).

[38] C. Savage, K. Freese, and P. Gondolo, Phys. Rev. D 74, 043531 (2006). This paper improves upon the Lewin-Smith calculation by properly including the effect of the solarsystem velocity on the cutoff at high WIMP velocity.

[39] R. Helm, Phys. Rev. 104, 1466 (1956).

[40] J. D. Lewin, P. F. Smith, Astropart. Phys. 6, 87 (1996).

[41] A. Manzur, A. Curioni, L. Kastens, D. N. McKinsey, K. Ni, and T. Wongjirad, Phys. Rev. C 81025808 (2010).

[42] P. Sorensen et al., Proc. Sci., IDM2010 (2011) 017.

[43] See the Supplemental Materials at http://link.aps.org/ supplemental/10.1103/PhysRevLett.112.091303 for additional plots and analysis details.

[44] C. McCabe, Phys. Rev. D 82023530 (2010).

[45] E. Armengaud et al., Phys. Lett. B 702, 329 (2011).

[46] Z. Ahmed et al., Science 327, 1619 (2010); R. Agnese et al., arXiv:0912.3592.

[47] D. Akimov et al., Phys. Lett. B 709, 14 (2012).

[48] R. Agnese et al., arXiv:1309.3259.

[49] M. Felizardo et al., Phys. Rev. Lett. 108, 201302 (2012).

[50] E. Aprile et al., Phys. Rev. Lett. 109, 181301 (2012).

[51] C. E. Aalseth et al., Phys. Rev. D 88, 012002 (2013).

[52] Z. Ahmed et al., Phys. Rev. Lett. 106, 131302 (2011).

[53] R. Agnese et al. (to be published).

[54] G. Angloher et al., Eur. Phys. J. C 72, 1971 (2012).

[55] R. Bernabei et al., Eur. Phys. J. C 56, 333 (2008).

[56] C. Savage, G. Gelmini, P. Gondolo, and K. Freese, J. Cosmol. Astropart. Phys. 04 (2009) 010.

[57] DM Tools, http://dmtools.brown.edu:8080/. 